\title{
EFICIÊNCIA DA SUPLEMENTAÇÃO ALIMENTAR EM TILÁPIAS DO NILO USANDO MODELO EXPONENCIAL DE CRESCIMENTO
}

\author{
EFFICIENCY OF FEED SUPPLEMENTATION FOR NILE TILAPIA \\ USING EXPONENTIAL GROWTH MODEL
}

\author{
Garcia, F. ${ }^{*}$, Santos, V.B. ${ }^{2}$ e Moraes, F.R. ${ }^{3}$
}

\begin{abstract}
${ }^{1}$ Secretaria de Agricultura e Abastecimento do Estado de São Paulo. Agência Paulista de Tecnologia dos Agronegócios, APTA. Pólo Regional do Noroeste Paulista. Votuporanga, SP. Brasil. *fgarcia@apta.sp.gov.br ${ }^{2}$ Secretaria de Agricultura e Abastecimento do Estado de São Paulo. Agência Paulista de Tecnologia dos Agronegócios, APTA. Pólo Regional da Alta Sorocabana. Presidente Prudente, SP. Brasil.

${ }^{3}$ Centro de Aqüicultura da Unesp. Universidade Estadual Paulista. Jaboticabal, SP. Brasil.
\end{abstract}

\section{PalaVRas chaVe adicionais}

Levedura. Oreochromis niloticus. Mananoligossacarídeo. $\beta$-glucano.

\section{RESUMO}

Objetivou-se avaliar a aplicabilidade do modelo de crescimento exponencial no estudo da eficiência da suplementação alimentar com mananoligossacarídeo e $\beta$-glucano para tilápias do Nilo criadas em tanques-rede. $O$ experimento foi realizado em piscicultura de criação de tilápias em tanques-rede. Tilápias jovens, com peso inicial de 25,4 $\pm 2,04 \mathrm{~g}$, foram distribuídas em 12 tanquesrede de $2 \times 2 \times 1,5 \mathrm{~m}$, com volume útil de $6 \mathrm{~m}^{3} \mathrm{e}$ densidade de 2500 peixes por tanque. Para estudo realizou-se a biometria individual de uma amostra aleatória de $5 \%$ dos peixes de cada unidade experimental. Os resultados permitem concluir que a adição de suplemento até o nível de $2000 \mathrm{mg} /$ $\mathrm{kg}$ de ração não altera a taxa de crescimento. $\mathrm{O}$ modelo exponencial pode ser usado para comparar a eficiência de diferentes dietas.

\section{SUMMARY}

The aim of this study was to evaluate the applicability of the exponential growth model to study feed supplementation efficiency with mannan oligosaccharide and $\beta$-glucan in Nile tilapia raised in net cages. The trial was carried out in a cage fish farm. Tilapia juveniles (initial weigh $25.4 \pm 2.04 \mathrm{~g}$ ) were distributed in 12 cages $(2 \times 2 \times 1.5 \mathrm{~m})$, with $6 \mathrm{~m}^{3}$ volume and stock density of 2500 fish per cage. To evaluate the exponential growth model,

Recibido: 2-2-08. Aceptado: 9-6-09.

\section{AdDitiOnAL KEYWORDS}

Yeast. Oreochromis niloticus. Mannan oligosaccharide. $\beta$-glucan.

a randomized sample of $5 \%$ fish was collected for individual biometry in each experimental unit. The results show that none of supplement levels increased the growth ratio of Nile tilapias and the exponential growth model can be used to compare efficiency of different diets.

\section{INTRODUÇÃO}

O Ministério da Pesca e Aquicultura (MPA) do governo brasileiro está normatizando a utilização das águas públicas da União, através da criação de parques aquícolas, que oficializarão uma opção econômica para o sistema intensivo em reservatórios de usinas hidroelétricas.

Os estudos relacionados à utilização de prebióticos, substâncias seletivamente fermentadas por microrganismos da flora gastrintestinal(Roy e Gibson, 1999), tornamse de grande importância no sistema de criação em tanques-rede. Gatlin III et al. (2006) destacam que os prebióticos, podem melhorar o desempenho produtivo, a eficiência alimentar e a resistência às enfermidades, com redução nos custos de produção e agregação de valor à imagem do 
produto aqüicola. Dentre estes suplementos alimentares, destacam-se os componentes de parede celular de levedura, os mananoligossacarídeos (MOS) e os $\beta$ glucanos, com propriedades prebióticas e imunoestimulantes, respectivamente (Welker et al., 2007).

$\mathrm{O}$ efeito destes produtos é mais bem evidenciado em situação de desafio sanitário (Mosenthin e Bauer, 2000). Portanto, as pesquisas realizadas em condições de laboratório muitas vezes não apresentam resultados conclusivos e aplicáveis por não representarem a realidade do ambiente de criação, onde os peixes estão submetidos a uma série de situações de estresse. Pensando-se na necessidade do desenvolvimento da pesquisa de campo, a piscicultura em tanques-rede apresenta uma estrutura bastante interessante para execução de ensaios por oferecer repetição e casualização dos tratamentos. Cada piscicultura é composta por um número grande de tanques-rede, com volume útil e densidade de estocagem semelhantes, inseridos em um ambiente comum, o que permite ao pesquisador o uso desses tanques como unidades experimentais (Garcia, 2007).

Como ferramenta de análise, pode-se estudar o crescimento de animais por meio do ajuste de funções não-lineares, pois, dessa maneira, é possível sintetizar informações de todo o período de vida, ou seja, um conjunto de informações em série de peso por idade, em um pequeno conjunto de parâmetros interpretáveis biologicamente, facilitando, assim, o entendimento do fenômeno (Oliveira et al., 2000; García et al., 1995). Santos et al. (2008) utilizaram o modelo exponencial para comparação do crescimento de linhagens de tilápia do Nilo criadas em tanques-rede e obtiveram sucesso. O objetivo deste estudo foi avaliar a aplicabilidade do modelo de crescimento exponencial no estudo da eficiência da suplementação alimentar com mananoligossacarídeo e $\beta$-glucano para tilápias do Nilo criadas em tanques-rede.

\section{MATERIAL E MÉTODOS}

O experimento foi realizado no reservatório de Nova Avanhandava, córrego Arribada, afluente do rio Tietê, no município de Zacarias, SP situado a latitude de $21^{\circ} 3^{\prime} 7^{\prime \prime}$ S e longitude $50^{\circ} 3^{\prime} 3^{\prime \prime} \mathrm{W}$.

Foram utilizadas tilápias do Nilo Oreochromis niloticus jovens, em bom estado fisiológico, com idade aproximada de 80 dias, revertidas sexualmente para machos, com peso inicial de $25,4 \pm 2,04 \mathrm{~g}$, distribuídas em 12 tanques-rede de $2 \times 2 \times 1,5 \mathrm{~m}$, com volume útil de $6 \mathrm{~m}^{3}$ e densidade de 2500 peixes por tanque.

A ração utilizada foi de formulação e processamento comerciais, de modo a atender as exigências da espécie estudada na primeira fase de criação (tabela I), contendo $40 \%$ de proteína bruta e extrusada no diâmetro de $2,6 \mathrm{~mm}$. O experimento foi conduzido em delineamento inteiramente casualisado, sendo que os tratamentos consistiram na inclusão de $0,500,1000$ e $2000 \mathrm{mg}$ do suplemento alimentar ${ }^{1}$, contendo $25 \%$ mananoligossacarídeo e $30 \% \beta$ glucano $/ \mathrm{kg}$ de ração, com três repetições (tanques-rede). A dieta foi oferecia ad libtum, quatro vezes ao dia, evitando-se sobras.

\section{VARIÁVEIS AQUÁTICAS}

As variáveis limnológicas foram mensuradas no local de inserção dos tanquesrede, sendo tomados os dados de temperatura, oxigênio dissolvido, transparência, amônia, nitrito e nitrato, $\mathrm{pH}$, material em suspensão, nitrogênio total, condutividade, fósforo total e clorofila. Todas as variáveis mantiveram-se dentro dos valores recomendados para a espécie em estudo (SipaúbaTavares, 1994): temperatura $22,1 \pm 0,9^{\circ} \mathrm{C}$, oxigênio dissolvido $7,19 \pm 1,28 \mathrm{mg} / 1$, saturação de oxigênio dissolvido $82,4 \pm$ $14,4 \%, \mathrm{pH} 7,72 \pm 0,53$, material em suspensão $1,3 \pm 1,2 \mathrm{mg} / 1$, nitrogênio total $0,51 \pm 0,34 \mathrm{mg} /$

${ }^{1}$ Active $\operatorname{mos}{ }^{\circledR}$ - Biorigin. 
Tabela I. Composição percentual básica das dietas experimentais. (Percentual composition of experimental diets).

\begin{tabular}{lc}
\hline Ingrediente & $\%$ \\
Milho moído & 8,46 \\
Farelo de trigo & 20,05 \\
Farelo soja & 11,10 \\
Glúten de milho & 15,00 \\
Quirera de arroz & 12,00 \\
Farinha de peixe & 11,00 \\
Farinha de vísceras de aves & 15,00 \\
NaCl & 0,25 \\
Vixil lignossulfonato-aglutinante & 1,00 \\
Farinha de pena & 5,00 \\
Suplemento vitamínico e mineral & 1,00 \\
Cloreto de colina 60\% & 0,05 \\
LD Metionina & 0,09 \\
& \\
Composição calculada & $(\%)$ \\
Proteína bruta & 40,00 \\
Extrato etéreo & 5,00 \\
Fibra bruta & 3,80 \\
Energia (kcal/kg) & 4160,40 \\
Cinzas & 8,44 \\
Ca & 1,8 \\
P & 1,28
\end{tabular}

Suplemento vitamínico e mineral (quantidade/kg de ração): $2000,00 \mathrm{mg}$ colina; $0,2374 \%$ sódio; $0,2709 \%$ cloro; $0,7186 \%$ potássio; $0,2463 \%$ magnésio; $0,4434 \%$ enxofre; $449,10 \mathrm{mg}$ ferro; $31,88 \mathrm{mg}$ cobre; $145,49 \mathrm{mg}$ manganês; $365,33 \mathrm{mg}$ zinco; $0,011 \mathrm{mg}$ cobalto; $9,52 \mathrm{mg}$ iodo; $0,912 \mathrm{mg}$ selênio; 27664,3 Ul vitamina A; 5962,81 Ul vitamina D3; 350 mg vitamina $E ; 11,93$ mg vitamina K3; 13,95 $\mathrm{mg}$ vitamina $B 1 ; 20,38 \mathrm{mg}$ vitamina $B 2 ; 17,31 \mathrm{mg}$ vitamina B6; 40,28 mcg vitamina B12; $263,79 \mathrm{mg}$ niacina; $48,11 \mathrm{mg}$ ácido pantotênico; $2,47 \mathrm{mg}$ ácido fólico; $0,38 \mathrm{mg}$ biotina; $400 \mathrm{mg}$ vitamina $\mathrm{C}$.

1, amônia $80,75 \pm 74,84 \mu \mathrm{g} / 1$, nitrito $1,30 \pm 1,01$ $\mu \mathrm{g} / \mathrm{L}$, nitrato $15 \pm 13,74 \mu \mathrm{g} / 1$, condutividade $170,7 \pm 5,6 \mu \mathrm{S} / \mathrm{cm}$, transparência $1,35 \pm 0,41$ $\mathrm{m}$, fósforo total $15,5 \pm 15 \mu \mathrm{g} / \mathrm{l}$ e clorofila 0,8 $\pm 0,5 \mu \mathrm{g} / \mathrm{l}$.

\section{Curvasde CRESCIMENTO}

Após 30 e 60 dias de alimentação com as dietas testadas, realizou-se a biometria indi- vidual de uma amostra aleatória de 5\% dos peixes de cada unidade experimental, totalizando 125 peixes por tanque. O estudo do crescimento foi realizado mediante o seguinte modelo da equação exponencial:

$$
y i=A e^{K x i}+e i
$$

sendo:

$y i=$ o peso observado de cada peixe, $i=1,2, \ldots, n$, $A=a$ estimativa do peso inicial,

$\mathrm{e}=\mathrm{a}$ base do logaritmo natural,

$\mathrm{K}=\mathrm{a}$ taxa de crescimento específico,

$\mathrm{xi}=\mathrm{o}$ tempo, ou a idade referente a cada peixe,

e $i=0$ erro associado a cada observação, que por pressuposição é $\mathrm{N}\left(0, \sigma^{2}\right)$.

Os parâmetros das curvas para cada nível de inclusão do suplemento foram comparados pelos seus intervalos de confiança com probabilidade de $95 \%$. Devido à existência de heterogeneidade de variâncias, uma vez que a variância dos pesos corporais aumenta muito com a idade, o inverso das variâncias dos pesos foi utilizado como fator de ponderação. Os ajustes foram obtidos através do procedimento MODEL do pacote computacional SAS para Windows versão 8.1, utilizando-se a opção WEIGHT e a macro $\% A R$, pois se constatou a existência de auto-relação residual pelo teste de DurbinWatson (Draper e Smith, 1998).

\section{RESULTADOSEDISCUSSÃO}

A tabela II apresenta os pesos (g) médios, mínimos e máximos observados em cada unidade experimental (tanque-rede), bem como os desvios padrão e os fatores de ponderação utilizados no ajuste do modelo nos diferentes tempos de cultivo.

A tabela III apresenta as estimativas dos parâmetros do modelo exponencial de crescimento das tilápias submetidas aos diferentes níveis de inclusão do suplemento na ração.

Pode-se observar que embora a estimativa do peso inicial (A) do tratamento controle tenha sido superior às demais, sua taxa 


\section{GARCIA, SANTOSEMORAES}

de crescimento $(\mathrm{K})(0,61 \mathrm{~g} / \mathrm{dia})$ foi inferior ao grupo alimentado com $2000 \mathrm{mg}$ do suple- mento/kg de ração $(0,71 \mathrm{~g} / \mathrm{dia})$, e essa diferença foi na ordem de $13,8 \%$, demons-

Tabela II. Pesos (g) médios, mínimos e máximos observados nos tanques, desvio padrão (S) dos pesos e fator de ponderação utilizado no ajuste do modelo exponencial. (Average weights (g), minimum and maximum weights observed in the cages, weight standard deviation (S) and weighting factor used on exponential model fit).

\begin{tabular}{|c|c|c|c|c|c|c|c|}
\hline $\begin{array}{l}\text { Suplemento } \\
(\mathrm{mg} / \mathrm{kg})\end{array}$ & Mês & Repetição & médio & $\begin{array}{l}\text { Peso }(g) \\
\text { mínimo }\end{array}$ & máximo & $\mathrm{S}$ & $\begin{array}{c}\text { Fator de } \\
\text { ponderação }\end{array}$ \\
\hline \multirow{9}{*}{0} & \multirow{4}{*}{1} & 1 & 29,39 & 8,00 & 70,00 & 13,99 & 0,005109 \\
\hline & & 2 & 44,16 & 4,00 & 84,00 & 21,74 & 0,002115 \\
\hline & & 3 & 44,72 & 12,00 & 104,00 & 21,86 & 0,002092 \\
\hline & & 1 & 69,93 & 14,00 & 148,00 & 31,61 & 0,001001 \\
\hline & \multirow[t]{3}{*}{2} & 2 & 100,35 & 10,00 & 198,00 & 46,96 & 0,000454 \\
\hline & & 3 & 96,54 & 14,00 & 250,00 & 47,11 & 0,000451 \\
\hline & & 1 & 111,99 & 26,00 & 230,00 & 47,37 & 0,000446 \\
\hline & \multirow[t]{2}{*}{3} & 2 & 141,36 & 16,00 & 316,00 & 65,09 & 0,000236 \\
\hline & & 3 & 130,91 & 26,00 & 300,00 & 65,88 & 0,000230 \\
\hline \multirow{9}{*}{500} & \multirow{4}{*}{1} & 1 & 28,01 & 8,00 & 60,00 & 12,33 & 0,006578 \\
\hline & & 2 & 27,34 & 4,00 & 58,00 & 11,52 & 0,007533 \\
\hline & & 3 & 39,60 & 8,00 & 94,00 & 18,56 & 0,002903 \\
\hline & & 1 & 65,74 & 12,00 & 126,00 & 29,31 & 0,001164 \\
\hline & \multirow[t]{3}{*}{2} & 2 & 65,94 & 6,00 & 150,00 & 32,34 & 0,000956 \\
\hline & & 3 & 96,56 & 30,00 & 168,00 & 32,06 & 0,000973 \\
\hline & & 1 & 109,88 & 32,00 & 242,00 & 46,77 & 0,000457 \\
\hline & \multirow[t]{2}{*}{3} & 2 & 110,77 & 20,00 & 216,00 & 41,82 & 0,000572 \\
\hline & & 3 & 114,82 & 18,00 & 258,00 & 57,91 & 0,000298 \\
\hline \multirow{9}{*}{1000} & \multirow{4}{*}{1} & 1 & 24,12 & 6,00 & 58,00 & 11,99 & 0,006951 \\
\hline & & 2 & 29,90 & 8,00 & 66,00 & 11,55 & 0,007492 \\
\hline & & 3 & 37,25 & 8,00 & 82,00 & 18,71 & 0,002858 \\
\hline & & 1 & 83,06 & 11,00 & 140,00 & 27,34 & 0,001338 \\
\hline & \multirow[t]{3}{*}{2} & 2 & 75,12 & 22,00 & 160,00 & 27,24 & 0,001348 \\
\hline & & 3 & 95,91 & 16,00 & 190,00 & 38,21 & 0,000685 \\
\hline & & 1 & 97,40 & 14,00 & 258,00 & 47,38 & 0,000445 \\
\hline & \multirow[t]{2}{*}{3} & 2 & 119,63 & 22,00 & 244,00 & 49,87 & 0,000402 \\
\hline & & 3 & 140,72 & 28,00 & 298,00 & 63,75 & 0,000246 \\
\hline \multirow{9}{*}{2000} & \multirow{4}{*}{1} & 1 & 32,76 & 4,00 & 96,00 & 21,16 & 0,002234 \\
\hline & & 2 & 34,47 & 4,00 & 98,00 & 21,24 & 0,002216 \\
\hline & & 3 & 23,52 & 4,00 & 60,00 & 11,22 & 0,007948 \\
\hline & & 1 & 93,15 & 14,00 & 230,00 & 41,69 & 0,000575 \\
\hline & \multirow[t]{3}{*}{2} & 2 & 91,04 & 14,00 & 192,00 & 43,06 & 0,000539 \\
\hline & & 3 & 61,00 & 14,00 & 146,00 & 27,95 & 0,001280 \\
\hline & & 1 & 127,59 & 16,00 & 316,00 & 66,41 & 0,000227 \\
\hline & \multirow[t]{2}{*}{3} & 2 & 143,44 & 20,00 & 286,00 & 63,23 & 0,000250 \\
\hline & & 3 & 92,98 & 14,00 & 252,00 & 47,57 & 0,000442 \\
\hline
\end{tabular}

Archivos de zootecnia vol. 60, núm. 232, p. 1242. 
Tabela III. Estimativas dos parâmetros do modelo exponencial, erro padrão e limites inferior e superior dos intervalos de confiança dos tratamentos. (Estimate parameters of exponential model, standard error and confidence limit of the treatments).

\begin{tabular}{|c|c|c|c|c|c|c|}
\hline \multirow{3}{*}{$\begin{array}{l}\text { Suplemento } \\
(\mathrm{mg} / \mathrm{kg})\end{array}$} & \multicolumn{2}{|c|}{ Parâmetros (erro padrão) } & \multicolumn{4}{|c|}{ Intervalo de confiança } \\
\hline & \multirow[t]{2}{*}{$A(g)$} & \multirow[t]{2}{*}{ K (g/dia) } & \multicolumn{2}{|c|}{ A } & \multicolumn{2}{|c|}{$\mathrm{K}$} \\
\hline & & & inferior & superior & inferior & superior \\
\hline $0 \mathrm{mg}$ & $20,8862^{A *}(0,9946)$ & $0,6128^{\mathrm{B}} \quad(0,0214)$ & 18,9128 & 22,8440 & 0,5718 & 0,6556 \\
\hline $500 \mathrm{mg}$ & $16,6250^{\mathrm{B}} \quad(0,7110)$ & $0,6576^{A B} \quad(0,0194)$ & 15,2658 & 18,0186 & 0,6197 & 0,6949 \\
\hline $1000 \mathrm{mg}$ & $16,1860^{\mathrm{B}} \quad(0,7397)$ & $0,6918^{A B} \quad(0,0210)$ & 14,7697 & 17,6240 & 0,6522 & 0,7323 \\
\hline $2000 \mathrm{mg}$ & $14,7404^{\mathrm{B}}(0,8305)$ & $0,7109^{A} \quad(0,0250)$ & 13,1556 & 16,3918 & 0,6629 & 0,7592 \\
\hline
\end{tabular}

*Estimativas seguidas de letras maiúsculas diferentes em uma mesma coluna, não são iguais pela sobreposição de intervalo de confiança a $5 \%$.

trando que a suplementação alimentar com o maior nível testado elevou a taxa de crescimento das tilápias criadas em tanques-rede.

Poderia-se esperar que o tratamento com $2000 \mathrm{mg} / \mathrm{kg}$ de MOS aumentasse a taxa de crescimento dos peixes. Entretanto, a diferença encontrada no peso inicial (parâmetro A) impossibilitou qualquer tipo de comparação entre as taxas de crescimento dos peixes recebendo os diferentes níveis de suplementação devido às relações existentes entre os parâmetros A e K.

O comportamento das curvas, bem como os coeficientes de determinação ajustados $\left(\mathrm{R}^{2} \mathrm{Aj}\right.$.) podem ser visualizados na figura 1 .

Estudo com sea bass Dicentrarchus labrax demonstraram que a adição de $200 \mathrm{e}$ $400 \mathrm{mg}$ de MOS/kg de ração elevou o ganho de peso dos peixes (Torrecillas et al., 2007). O mecanismo de ação do MOS é a melhora na utilização de nutrientes da dieta por atuar na redução da carga de bactérias intestinais indesejáveis e aumentar a área de absorção da mucosa (Moran, 2004). O MOS possui o açúcar manana que é reconhecido pelos patógenos, mas são indisponíveis para uso quando estão ligados à forma de oligossacarídeo. Os patógenos são atraídos a se ligarem ao oligossacarídeo e então, tornamse incapazes de aderirem à mucosa intestinal (McCann et al., 2006). Como observado em estudo complementar a este, verificouse resposta positiva na integridade das vilosidades intestinais de tilápias alimentadas com 500, 1000 e $2000 \mathrm{mg}$ deste composto por $\mathrm{kg}$ de ração durante 37 dias, com conseqüente melhora na digestibilidade da proteína da ração (Garcia, 2008).

Por outro lado, em ensaio realizado em laboratório (Garcia, 2008), com densidade de estocagem reduzida e condições ambientais controladas, esta resposta não foi observada. O que demonstra que o nível de estresse do animal e o ambiente onde é

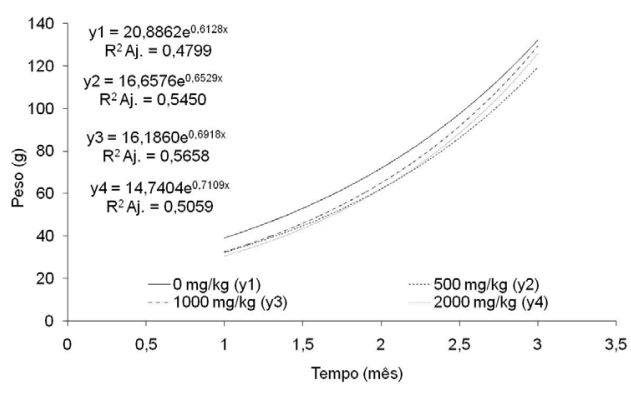

Figura 1. Peso (g) em função do tempo do modelo exponencial de crescimento de tilápias para cada nível de inclusão do suplemento testado (0, 500, 1000 e $2000 \mathrm{mg}$ / $\mathrm{kg}$ ). (Weight $(\mathrm{g})$ in function of time following the exponential growth model of tilapias in each level of the tested supplementation $(0,500,1000$ and $2000 \mathrm{mg} / \mathrm{kg}$ ). 


\section{GARCIA, SANTOSEMORAES}

criado têm relação direta com a resposta biológica obtida pela adição de prebióticos à dieta, pois em condições não estressantes, supõe-se que a microbiota intestinal esteja em condição de equilíbrio, ou seja, com ou sem o fornecimento de prebióticos as respostas obtidas serão semelhantes. No entanto, quando em condição de estresse, o efeito benéfico do fornecimento de prebióticos sobre a resposta biológica é evidenciado (Mathew et al., 1993; Mosenthin e Bauer, 2000). Estes resultados ressaltam a importância da execução de experimentos de campo em situações reais de estresse para testes de uso de suplementos alimentares.

Por se tratar de um experimento de campo, optou-se por fazer a comparação das curvas de crescimento das repetições dentro de cada tratamento (tabela IV e figura 2) com o objetivo de verificar a confiabilidade dos resultados e se as variáveis não controláveis presentes na piscicultura estariam interferindo no experimento. Verifica-se que, embora houvesse diferença no peso inicial dos animais das repetições dentro de cada tratamento, não se verificou diferença entre a taxa de crescimento $\mathrm{K}$ dos peixes exceto no grupo de que recebeu 500 $\mathrm{mg}$ do suplemento/kg de ração.

Segundo Bastianelli e Sauvant (1997), diversos fatores podem influenciar a curva de crescimento, dentre eles, a fase de cultivo (idade do peixe), espécie estudada, ambiente de cultivo, densidade de estocagem, variáveis limnológicas e fatores relacionados à alimentação: qualidade do alimento, freqüência da alimentação, quantidade oferecida, dentre outras. Desta forma, a ausência de diferença entre as repetições

Tabela IV. Estimativas dos parâmetros do modelo exponencial, erro padrão e limites inferior e superior dos intervalos de confiança das repetições para os diferentes níveis de inclusão do suplemento alimentar. (Estimate parameters of the exponential model, standard error and confidence limits of repetitions to the different levels of feed supplement).

\begin{tabular}{|c|c|c|c|c|c|c|c|c|}
\hline \multirow[b]{2}{*}{$\begin{array}{c}\text { Suplemento } \\
\mathrm{mg} / \mathrm{kg}\end{array}$} & \multirow[b]{2}{*}{ Repetição } & \multicolumn{2}{|c|}{ Parâmetros (erro padrão) } & \multicolumn{4}{|c|}{ Intervalo de confiança } & \multirow[t]{2}{*}{$R^{2} \mathrm{Aj}$} \\
\hline & & A & $\mathrm{K}$ & inferior & $\begin{array}{l}\text { A } \\
\text { superior }\end{array}$ & inferior & $\begin{array}{l}\mathrm{K} \\
\text { superior }\end{array}$ & \\
\hline \multirow{3}{*}{0} & 1 & $16,03^{\mathrm{B}}(1,17)$ & $0,66^{A}(0,03)$ & 13,78 & 18,33 & 0,60 & 0,73 & 0,5582 \\
\hline & 2 & $26,56^{A}(1,86)$ & $0,5^{A}(0,03)$ & 22,96 & 30,21 & 0,52 & 0,64 & 0,4660 \\
\hline & 3 & $27,74^{\mathrm{A}}(1,92)$ & $0,53^{A}(0,03)$ & 24,06 & 31,52 & 0,48 & 0,60 & 0,4079 \\
\hline \multirow{3}{*}{500} & 1 & $14,85^{\mathrm{B}}(0,88)$ & $0,68^{\mathrm{A}}(0,02)$ & 13,11 & 16,59 & 0,63 & 0,74 & 0,5542 \\
\hline & 2 & $14,19^{\mathrm{B}}(0,85)$ & $0,69^{A}(0,02)$ & 12,53 & 15,88 & 0,64 & 0,75 & 0,5993 \\
\hline & 3 & $26,21^{\mathrm{A}}(1,95)$ & $0,54^{\mathrm{B}}(0,03)$ & 22,60 & 30,05 & 0,47 & 0,61 & 0,4738 \\
\hline \multirow{3}{*}{1000} & 1 & $14,50^{\mathrm{B}}(1,31)$ & $0,69^{A}(0,04)$ & 12,13 & 17,09 & 0,61 & 0,77 & 0,5693 \\
\hline & 2 & $15,88^{\mathrm{B}}(0,86)$ & $0,69^{A}(0,02)$ & 14,20 & 17,58 & 0,65 & 0,75 & 0,5980 \\
\hline & 3 & $21,34^{A}(1,60)$ & $0,65^{A}(0,03)$ & 18,32 & 24,49 & 0,59 & 0,72 & 0,5414 \\
\hline \multirow{3}{*}{2000} & 1 & $19,62^{\mathrm{A}}(1,85)$ & $0,65^{A}(0,04)$ & 16,20 & 23,27 & 0,58 & 0,74 & 0,4811 \\
\hline & 2 & $19,13^{A}(1,59)$ & $0,68^{A}(0,03)$ & 16,07 & 22,27 & 0,62 & 0,76 & 0,5298 \\
\hline & 3 & $12,65^{\mathrm{B}}(0,89)$ & $0,69^{A}(0,03)$ & 10,96 & 14,41 & 0,63 & 0,75 & 0,5017 \\
\hline
\end{tabular}

*Estimativas seguidas de letras maiúsculas diferentes em uma mesma coluna, não são iguais pela sobreposição de intervalo de confiança a $5 \%$.

Archivos de zootecnia vol. 60, núm. 232, p. 1244. 


\section{MODELO DE CRESCIMENTO EXPONENCIAL PARA TILÁPIAS DO NILO}

demonstra que o ambiente da piscicultura em tanque-rede mostra-se adequado à experimentação, pois apesar de possuir inúmeras variáveis ambientais incontroláveis, estas se aplicam a todas as unidades experimentais de forma semelhante, de modo que, a diferença na taxa de crescimento observada deve-se, exclusivamente, aos diferentes níveis de suplementação a que os peixes foram submetidos.

Desta forma, pelo modelo de curvas exponenciais é possível comparar tratamentos. Entretanto, comparações nas taxas de crescimento são possíveis somente quando não se encontram diferenças na estimativa do peso inicial (A). Santos et al.
(2008) confirmam a aplicabilidade deste modelo no estudo de comparação de linhagens de tilápia do Nilo criadas em tanques-rede.

\section{CONCLUSÃO}

Os resultados do presente estudo permitem concluir que a adição de suplemento até o nível de $2000 \mathrm{mg} / \mathrm{kg}$ de ração não incrementa a taxa de crescimento de tilápias do Nilo criadas em sistema de tanques-rede e que o modelo de curvas exponenciais pode ser usado para comparar a eficiência de diferentes dietas em estudos de nutrição de peixes.
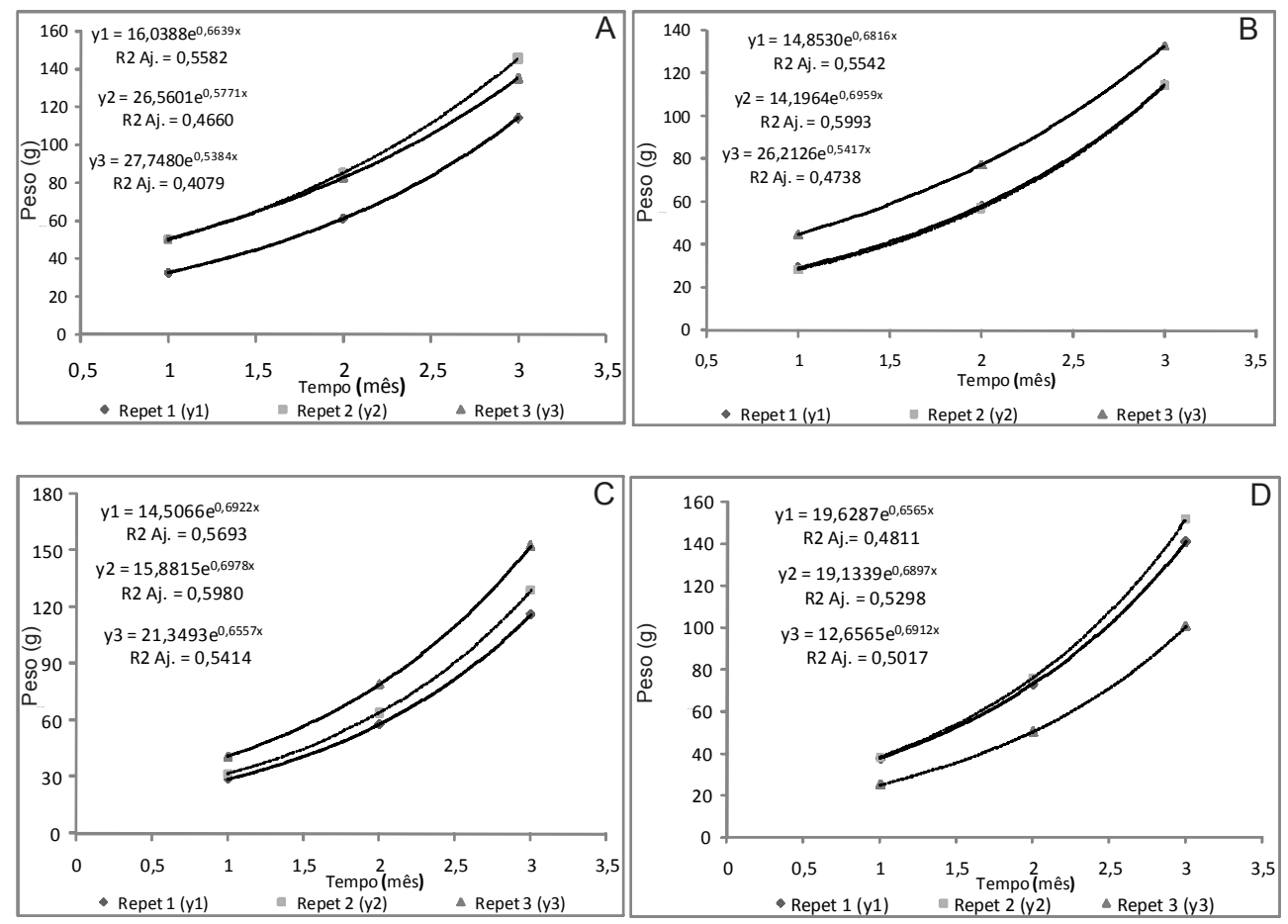

Figura 2. Peso ( $g$ ) em função do tempo do modelo exponencial de crescimento de tilápias nas diferentes repetições (tanques) em cada nível de inclusão do suplemento testado: $A$ - 0 $\mathrm{mg}$ do suplemento/kg de ração; B- $500 \mathrm{mg}$ do suplemento/kg de ração; $C$ - $1000 \mathrm{mg}$ do suplemento/kg de ração e D- $2000 \mathrm{mg}$ do suplemento/kg de ração. (Weight in function of time, following the exponential growth model, of tilapias in the different repetitions (cages) in each level of tested supplementation: A- 0 mg/kg of ration; B- $500 \mathrm{mg} / \mathrm{kg}$; C- $1000 \mathrm{mg} / \mathrm{kg}$ and D- $2000 \mathrm{mg} / \mathrm{kg}$ ). 


\section{GARCIA, SANTOSEMORAES}

\section{BIBLIOGRAFIA}

Bastianelli, D. e Sauvant, D. 1997. Modelling the mechanisms of pig growth. Livest. Prod. Sci., 51: 97-107.

Draper, N.R. and Smith, H. 1998. Applied regression analysis, $3^{\text {rd }}$ ed. Wiley. New York. 706 pp.

García, M.E.B., Alcalá, A.M., Moya, A.J.L., Bermejo, J.V.D. y Torre, F.S. 1995. Estimación de la curva de crecimiento en vacuno retinto desde el nacimiento hasta el destete. Arch. Zootec. 44: 179-192.

Garcia, F. 2007. Piscicultura em tanques-rede e a pesquisa de campo. Disponível em ftp:// ftp.sp.gov.br/ftppesca/piscicultura_tanques_ rede.pdf

Garcia, F. 2008. Suplementação alimentar com $\beta$ glucano e mananoligossacarídeo para tilápias do Nilo em tanques-rede. Tese de doutorado em Aquicultura. Caunesp. Unesp. Jaboticabal. 100 pp.

Gatlin III, D.M., Li, P., Wang, X., Burr, G.S., Castille, F. and Lawrence, A.L. 2006. Potential application of prebiotics in aquacuclture. En: Avances en Nutrición Acuícola VIII. Simposium Internacional de Nutrición Acuícola. Universidad Autónoma de Nuevo León. Monterrey. Nuevo León. México. pp. 371-376.

McCann, M.E.E., Newell, E., Preston, C. and Forbes, K. 2006. The use of mannan-oligosaccharides and/or tannin in broiler diets. Int. J. Poultry Sci., 5: 873-879.

Mathew, A.G., Sutton, A.L., Scheidt, A.B., Patterson, J.A., Kelly, D.T. and Meyerholtz, K.A. 1993. Effect of galactan on selected microbial populations and $\mathrm{pH}$ and volatile fatty acids in the ileum of the weanling pig. J. Anim. Sci., 71: 1503-1509.

Moran, C.A. 2004. Functional components of the cell wall of Saccharomyces cerevisiae: applications for yeast glucan and mannan. In: International feed industry symposium, 20. Proceedings... Alltech. Lexington. pp. 280-296. Mosenthin, R. and Bauer, E. 2000. The potential use of prebiotics in pig nutrition. In: International symposium on recent advances in animal nutrition. Proceedings... Seoul National University. Seoul. pp. 515-528.

Oliveira, H.N., Lôbo, R.B. e Pereira, C.S. 2000. Comparação de modelos não lineares para descrever o crescimento de fêmeas da raça Guzerá. Pesqui. Agropecu. Bras., 35: 1843-1851.

Roy, M. and Gibson, G.R. 1999. Probiotics and prebiotics. Microbal in menu. http://www. babelfish.altavista.com/cgi-bm (21/11/99).

Santos, V.B., Yoshihara, E., Freitas, R.T.F. and Reis Neto, R.V. 2008. Exponential growth model of Nile tilapia (Oreochromis niloticus) strains considering heteroscedastic variance. Aquaculture, 274: 96-100.

Sipauba Tavares, L.H. 1994. Limnologia aplicada a aqüicultura. FUNEP. Jaboticabal. 70 pp.

Torrecillas, S., Makol, A., Caballero, M.J., Montero, D., Robaina, L., Real, F., Sweetman, J., Tort, L. and Izquierdo, M.S. 2007. Immune stimulation and improved infection resistance in European sea bass (Dicentrarchus labrax) fed mannan oligosaccharides. Fish Shellfish Immun., 23: 969-981.

Welker, T.L., Lim, C., Yildirim-Aksoy, M., Shelby, R. and Klesius, P.H. 2007. Immune response and resistance to stress and Edwardsiella ictaluri challenge in channel catfish, Ictalurus punctatus, fed diets containing commercial whole cell yeast or yeast subcomponents. J. Aquacult. Soc., 38: 24-32.

Archivos de zootecnia vol. 60, núm. 232, p. 1246. 